\title{
Cavum septum pellucidum and obstructive hydrocephalus
}

\author{
P L Silbert, S S Gubbay, R J Vaughan
}

\begin{abstract}
Five patients presented with symptoms related directly to pressure effects from their cavum septum pellucidum with persistent or intermittent obstructive hydrocephalus. The most characteristic presenting symptoms were intermittent postural headache and postural loss of consciousness. If cysts of the cavum septum pellucidum are symptomatic and stereotactic cyst puncture or fenestration are ineffective, ventriculoperitoneal shunting should be carried out before resorting to more radical excision of the cyst.
\end{abstract}

$(\mathcal{F}$ Neurol Neurosurg Psychiatry 1993;56:820-822)

The presence of a cavum septum pellucidum represents a normal anatomical variant which is usually asymptomatic. Its prevalence in childhood declines with age, being present at necropsy in all premature infants, $85 \%$ of one month old infants, and $12-15 \%$ of six month old infants. In adults the prevalence varies significantly depending on the criteria used to diagnose a cavum septum pellucidum as some remnant should be present in all patients. ${ }^{1}$ In a recent series 14 out of 1914 adults $(0.73 \%)$ showed CT evidence of a cavum septum pellucidum. Five of these were boxers, who have a recognised increased incidence of cavum septum pellucidum. ${ }^{2}$

The septum pellucidum extends between the anterior portion of the corpus callosum and the body of the fornix. The cavum septum pellucidum and the more posterior cavum vergae are potential cavities that lie between the leaflets of the septum pellucidum. As a potential cavity, they are a normal finding. Controversy has existed for many years about whether enlargement of these cavities is responsible for any disease state. Many symptoms and diseases have been attributed to cysts of the septum pellucidum, but a cavum septum pellucidum is such a common finding that its previous associations are being questioned. Earlier series were biased because pneumoencephalography and necropsy represented the only available diagnosed modalities, $^{3}$ and descriptions of the septum pellucidum syndrome, characterised by mental disturbances, ataxia, disordered speech, epilepsy, and bilateral pyramidal signs, are now being questioned. ${ }^{4}$

We present a series of five patients (three of whom have been previously reported on ${ }^{5}$ ) who presented because of symptoms related directly to pressure effects from their cavum septum pellucidum.

Table Summary of clinical details and results of treatment and follow up in five cases of cavum septum pellucidum

Royal Perth Hospital, Box X2213, GPO,

Perth, Western Australia, 6001, Australia Department of Neurology P L Silbert S S Gubbay

Department of

Neurosurgery

R J Vaughan

Correspondence to: Professor Gubbay

Received 11 March 1992 and in revised form 27 August 1992 .

Accepted

11 September 1992

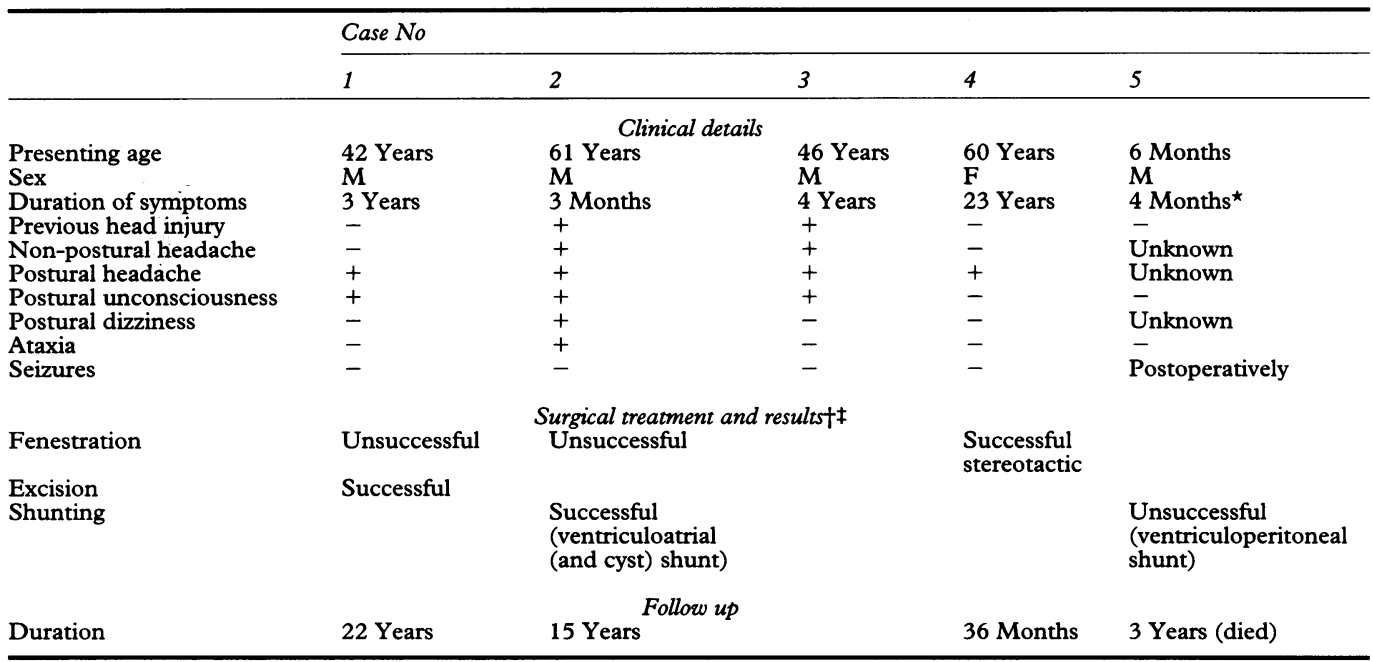

*Presented with delayed milestones at 6 months of age.

†Case 3 was lost to follow up.

Not all procedures were performed on all patients. 


\section{Case reports}

The case histories are summarised in the table. Cases 1 to 4 were of four adult patients presenting with the common symptom of headache between the ages of 42 and 61 years. In two instances there was a history of closed head injury with total recovery. The patients in cases 1 and 3 had experienced loss of consciousness when lying supine. The patient in case 2 became dizzy on lying supine and had occasionally lost consciousness when bending forward.

The patient in case 5 presented in infancy with delayed milestones of development and hydrocephalus which failed to respond to ventriculoperitoneal shunting because of continued distension of the cyst and possible shunt malfunction. No further motor or social development occurred and he died at 3 years of age of bronchopneumonia. The true cause of his condition only became evident at necropsy (figure: left).

Stereotactic puncture of the cyst was successful treatment in case 4 . Case 1 was initially treated by fenestration of the cyst but symptoms recurred and at reoperation the fenestrated walls of the cyst were found to be covered by a fine arachnoid membrane. The cyst walls were then excised with no recurrence of symptoms. In case 2 wide fenestration of the walls of the cyst resulted in only a temporary amelioration of symptoms, but ventriculoatrial shunting (with passage of the drainage catheter through the cavum septum pellucidum) was effective.

\section{Discussion}

The cavum septum pellucidum and the more posterior (often interconnecting) cavum vergae are often incorrectly called the fifth and sixth ventricles. They are not, however, part of the ventricular systems as they have a different embryological origin and are not lined by ependymal or choroid plexus cells. ${ }^{3}$ The cavum is a potential cavity and therefore significant enlargement is best termed a cyst of the cavum septum pellucidum. It is an important part of the limbic system, with prominent connections in both the medial (or Papez) limbic circuit and the basolateral limbic circuit. This provides direct and indirect afferent and efferent connections to the hippocampus, cingulate gyrus, medial frontal cortex, anterior nucleus of the thalamus, mamillary bodies, hypothalamus, and amygdaloid nuclei. ${ }^{3}$ Disease of the cavum septum pellucidum could therefore be expected to cause symptoms either by mass effect or by disturbance of the emotional and behavioural functions of the limbic system.

Cysts of the cavum septum pellucidum have been classified into two major groups. ${ }^{1}$ Incidental (asymptomatic) cava are seen in almost $1 \%$ of cranial CTs. ${ }^{2}$ Pathological (symptomatic) cava are comparatively rare, are always non-communicating, and may be associated with other structural or inflammatory brain disease.

Since the first cases reported by Dandy of an adult and a child with hydrocephalus due to a cyst of the cavum septum pellucidum (and vergae) ${ }^{6}$ further cases in adults, children, and infants have been reported occasionally, usually in single case reports or reviews. ${ }^{17-11}$ We have described five patients with symptoms of persistent or intermittent obstructive hydrocephalus associated with an enlarged cavum septum pellucidum. The most common presenting symptoms were intermittent headache (all four adult cases) and loss of consciousness (three of the four adult cases), presumably due to sudden transient obstruction of the foramina of Monro. In the more recent patients who underwent magnetic resonance imaging and pathological examination respectively (cases 4 and 5; fig) the foramina of Monro were patent. Amin has proposed a mechanism whereby intermittent obstruction of the foramina of Monro may occur. ${ }^{11}$ After foraminal obstruction the ventricles expand, causing alteration in the shape of the cyst and release of the obstruction. All four of the adult cases had a distinct postural component

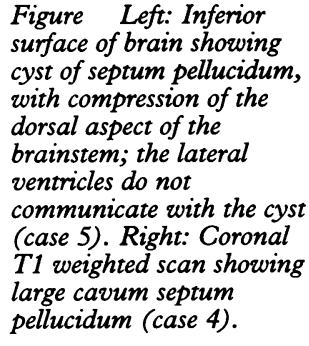

Figure Left: Inferior surface of brain showing with compression of the dorsal aspect of the brainstem; the lateral ventricles do not communicate with the cyst (case 5). Right: Coronal large cavum septum pellucidum (case 4)
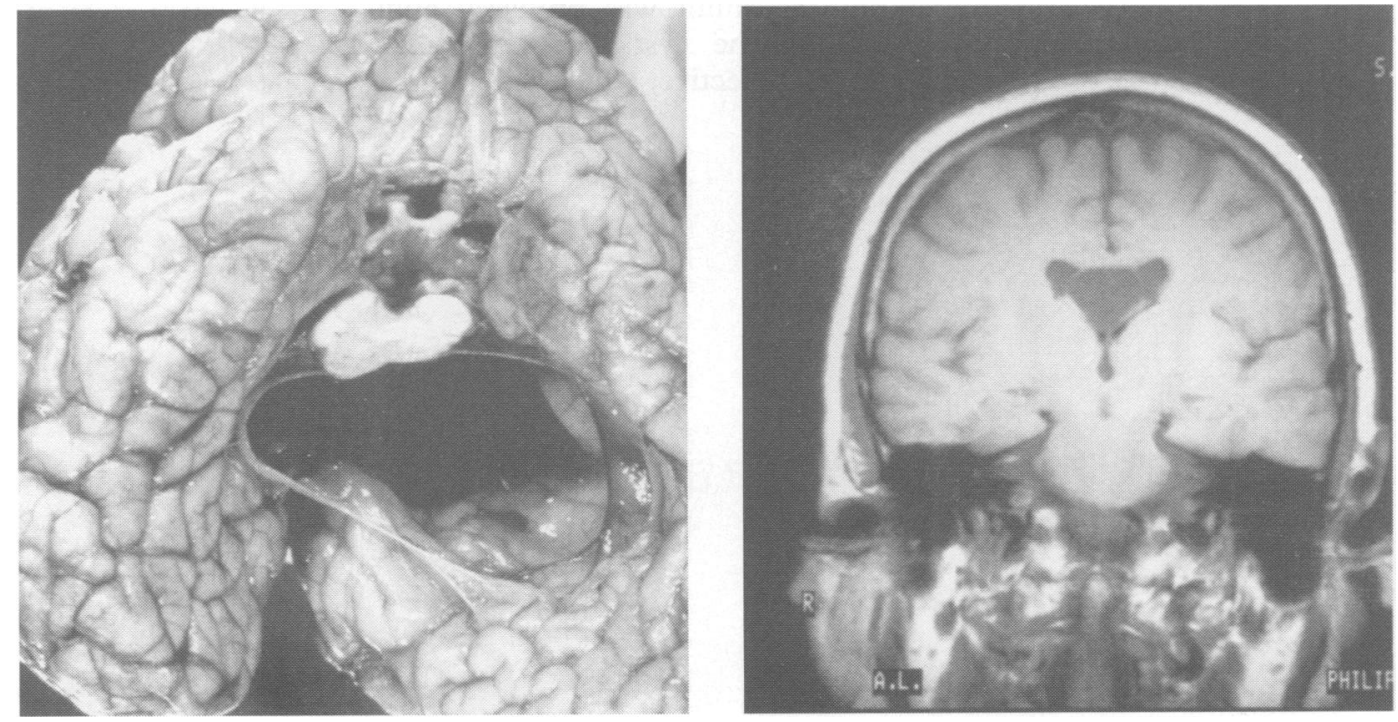
to their headache and in three of these patients a colloid cyst of the third ventricle was therefore the provisional diagnosis on initial clinical assessment. Whereas the patients in cases 1 and 2 were investigated before CT became available the response to surgery strongly supports the causative relation between the cyst of the cavum septum pellucidum and the patient's symptoms. In case 4 the history was suggestive and neuroimaging that clearly showed a large cavum septum pellucidum without ventricular dilatation (figure: right). Stereotactic puncture of the cyst of the cavum septum pellucidum produced a sustained remission from further headaches.

The patient in case 5 had severe hydrocephalus due to a combination of obstruction of the lateral ventricles and mass effect of the cyst of the cavum septum pellucidum (figure: left). The final recognition of the contribution of the cyst of the cavum septum pellucidum to his hydrocephalus was unfortunately only made at necropsy. With more advanced current neuroimaging techniques earlier diagnosis and appropriate therapy should now be possible.

The possible contribution of enlargement of the cavum to either psychiatric disturbances or epilepsy is controversial. None of our adult patients showed psychiatric abnormalities, behavioural changes, or epilepsy preoperatively, although postoperatively one patient (case 1) was confused and depressed, and another (case 2) confused with a transient left hemiparesis post-operatively. Earlier series evaluating the psychiatric aspects of the cavum septum pellucidum were subject to appreciable referral bias, ${ }^{3}$ but several recent reports describe possible associations with psychosis $^{1213}$ and a reversible Korsakoff's psychosis was found after a cyst of the septum pellucidum was excised. ${ }^{14}$

Craniotomy with fenestration (and biopsy) may not be as effective as shunting procedures in treating symptomatic cavum septum pellucidum. Stereotactic puncture is less invasive but would be liable to the same mechanism of potential failure as open fenestration. The child who died of progressive hydrocephalus did not respond to ventricular shunting, but the shunt was probably only partially draining the cyst and ventricular drainage was not effective, which led to pro- gressive hydrocephalus. Cavum septum pellucidum has been previously described as a cause of shunt malfunction. ${ }^{15}$

In conclusion, symptomatic enlargement of the cavum septum pellucidum is rare and most commonly causes intermittent obstructive hydrocephalus with headache and loss of consciousness; it is amenable to surgical treatment such as cyst puncture or shunting, ventriculoperitoneal shunting, or radical excision.

We thank Drs J B Stokes, D A Prentice, T Welborn, and $M$ Adonis for referring the patients. The surgery in cases 1 and 2 were performed by the late Mr J S Lekias, and in cases 4 and 5 by Mr M Lee. Postmortem examination and photography in case 5 was performed at the Department of Pathology, Princess Margaret Hospital. Other illustrations were provided by the Department of Medical Illustrations, Royal Perth Hospital, and Department of Medical Illustrations, Sir Charles Gairdner Hospital. Magnetic resonance imaging was performed by $\mathrm{Dr}$ Mark Khangure, Sir Charles Gairdner Hospital. We are grateful to the editor and publishers of Clinical and Experimental Neurology for permission to include cases $1-3 .^{5}$

1 Shaw CM, Alvord EC. Cava septi pellucidi et vergae: their normal and pathological states. Brain 1969;92: 213-24.

2 Bogdanoff B, Natter HM. Incidence of cavum septum pellucidum in adults: a sign of boxer's encephalopathy. pellucidum in aduits: a sign

3 Bruyn GW. Agenesis septi pellucidi, cavum septi pellucidi, cavum vergae, and cavum veli interposti. In: Vinken PJ, Bruyn GW, eds. Handbook of clinical neurology,
Vol 30. Amsterdam: North Holland Publishing

4 Wilder J. Hydrops des Cavum septi pellucidi. Klin Wochenschr 1938;17:414

5 Gubbay SS, Vaughan R, Lekias JS. Intermittent hydrocephalus due to cysts of the septum pellucidum: a study of three cases. Clinical and Experimental Neurology 1978; 14:93-9.

6 Dandy WE. Congenital cerebral cysts of the cavum septi pellucidi (fifth ventricle) and cavum vergae (sixth ventricle). Archives of Neurology and Psychiatry (Chicago) 1931;25:44-66.

7 Dooling EC, Barlow JF, Murphy JV, Richardson EP. Cyst of the cavum septi pellucidi. Arch Neurol 1972;27: of the

8 Heiskanen $O$. Cyst of the septum pellucidum causing increased intracranial pressure and hydrocephalus. f Neurosurg 1973;38:771-3.

9 Cowley AR, Moody DM, Alexander E, Ball MR, Laster DW. Distinctive CT appearance of cyst of the cavum septi pellucidi. $A \mathcal{F}$ 1979;133:548-50.

10 Garza-Mercado R. Giant cyst of the septum pellucidum. ₹ Neurosurg 1981;55:646-50.

11 Amin BH. Symptomatic cyst of the septum pellucidum. Child's Nerv Syst 1986;2:320-2.

12 Lewis SW, Mezey GC. Clinical correlates of septum pellucidum cavities: an unusual association with psychosis. Psychol Med 1985;15:43-54.

13 Mizukami K, Ofuku K, Shiraishi H, Koizumi J. Neuropsychiatric studies of the "pellucidum syndrom". $¥ p n \mathcal{F}$ psychiatric studies of the "pellucidu
Psychiatry Neurol 1989;43:67-75.

14 Gil Neciga E, Gil Peralta A, Polaina M, Sureda B, Bautista J. Cyst of the septum pellucidum and Bautista J. Cyst of the septum pellucidum
Korsakoff's psychosis. Eur Neurol 1989;29:99-101.

15 Mapstone TB, White RJ. Cavum septi pellucidi as a cause of shunt dysfunction. Surg Neurol 1981;16:96-8. 